Kansas State University Libraries

New Prairie Press

\title{
ANOTHER LOOK AT NEW GMA ORTHOGONAL ARRAYS
}

Yingfu Li

Timothy Wittig

Follow this and additional works at: https://newprairiepress.org/agstatconference

Part of the Agriculture Commons, and the Applied Statistics Commons

\section{(c) $(1) \ominus$}

This work is licensed under a Creative Commons Attribution-Noncommercial-No Derivative Works 4.0 License.

\section{Recommended Citation}

Li, Yingfu and Wittig, Timothy (2001). "ANOTHER LOOK AT NEW GMA ORTHOGONAL ARRAYS,"

Conference on Applied Statistics in Agriculture. https://doi.org/10.4148/2475-7772.1220

This is brought to you for free and open access by the Conferences at New Prairie Press. It has been accepted for inclusion in Conference on Applied Statistics in Agriculture by an authorized administrator of New Prairie Press. For more information, please contact cads@k-state.edu. 


\title{
ANOTHER LOOK AT NEW GMA ORTHOGONAL ARRAYS
}

\author{
YINGFU LI and TIMOTHY WITTIG
}

South Dakota State University

\begin{abstract}
Non-regular factorial designs have not been advocated until last decade due to their complex aliasing structure. However, some researchers recently found that the complex aliasing structure of non-regular factorial designs is a challenge as well as an opportunity. Li, Deng, and Tang (2000) studied non-regular designs and generated a collection of non-equivalent orthogonal arrays using a generalized minimum aberration criterion, proposed by Deng and Tang (1999). Some new orthogonal arrays they found cannot be embedded into Hadamard matrices. In this paper, we study these orthogonal arrays from the angle of projection. We show that these new GMA orthogonal arrays are also superior to the top designs obtained from Hadamard matrices when evaluated by the criteria of model estimability and design efficiency.
\end{abstract}

Key words and phrases: non-regular design, generalized minimum aberration, model estimability, design efficiency, Hadamard matrices, orthogonal arrays. 


\section{Introduction}

Two-level fractional factorial designs are most commonly used in practice. These designs can be generally classified into two broad categories: regular designs and non-regular designs. Regular designs, found in most classic textbooks on experimental designs, have two characteristics: their run sizes must be the power of 2 and their confounding patterns among the effects are completely determined by their defining relations. In contrast to regular designs, non-regular designs have flexible run size of multiples of 4 , but their confounding patterns are very complex. Besides the advantage of flexible run size, non-regular designs have been showed that their complex confounding relations can be exploited to detect some interaction effects, which would otherwise have been missed if regular designs had been used.

Non-regular designs used in practice and research are almost all chosen from Hadamard matrices due to their good properties. A Hadamard matrix $H$ of order $n$ is an $n \times n$ matrix with entries -1 and +1 such that the product of the transpose of $H$ and $H$ itself is $n$ times the identity matrix. However, two issues naturally arise in association with selecting fractional factorial designs from Hadamard matrices. First, there exist fractional factorial designs that cannot be embedded into Hadamard matrices. Vijayan (1976) studied the embedding problem of Hadamard matrices and showed that a fractional factorial design can be embedded into a Hadamard matrix if there are at most four missing columns. He further conjectured that if the number of factors $m \geq n / 2-1$, then the fractional factorial design can be embedded into a Hadamard matrix. However, counter-examples in Li, Deng and Tang (2000) disproved his conjecture. Second, the complete set of non-equivalent Hadamard matrices is only available for order 28 or lower. In fact, for orders $16,20,24$, and 28 , the numbers of non-equivalent Hadamard matrices are 5, 3, 60, and 487, respectively (Spence, 1995). By equivalent Hadamard matrices (or designs), we mean that one Hadamard matrix (or design) can be obtained from the other by permuting rows, permuting columns, switching the signs of a column, or a combination of these ways. It is not difficult to see that it is impractical to examine all of these non-equivalent Hadamard matrices even if the complete set were available for higher order.

Li, Deng and Tang (2000) studied fractional factorial designs obtained from orthogonal arrays and found that some top fractional factorial designs, ranked by the criterion of generalized minimum aberration, cannot be embedded into Hadamard matrices. By an orthogonal array with two-level " +1 " and " 1 ", we mean an $n \times m$ matrix with entries +1 and -1 such that the occurences of four level combinations $(+1,+1),(+1,-1),(-1,+1)$ and $(-1,-1)$ in every two columns of the matrix are the same. If we mathematically treat each column as a vector, then the dot product of any two column vectors in an orthogonal array is zero. An orthogonal array can be regarded as a (fractional) factorial design consisting of $n$ treatment combinations with $m$ factors. Therefore, we will speak of orthogonal arrays and factorial designs interchangeably in this paper.

In this paper, we study those top orthogonal arrays that cannot be embedded into Hadamard matrices from the angle of projection. We show that these new orthogonal arrays are superior to the top designs obtained from Hadamard matrices when further evaluated by the criteria of model estimability and design efficiency. For simplicity, we will focus on 
designs with 20 runs. Section 2 provides a brief review of design selecting criteria such as generalized minimum aberration and its related confounding frequency vector, design efficiency, and model estimability. The results of comparing the new designs with those obtained from Hadamard matrices are given in Section 3.

\section{Design selecting criteria}

One important problem in (screening) experimental designs is how to choose a good design given the number of factors $m$ and the run size $n$. For regular factorial designs, Box and Hunter (1961) introduced resolution, a concept that compares and assesses designs by the occurences of worst confounding. Fries and Hunter (1980) proposed a minimum aberration criterion to further distinguish two designs with same resolution by using the concept of the word-length pattern. The run size of a regular design must be a power of two and the defining relations of the regular design completely specify the aliasing pattern.

Non-regular factorial designs have a complex aliasing structure but a flexible run size of multiples of 4 . Some researchers recently have found that the complex aliasing structure of non-regular factorial designs is a challenge as well as an opportunity. For example, Hamada and Wu (1992) showed that the complex aliasing structure of Plackett and Burman designs can be exploited to detect some interaction cffects that would otherwise have been missed if regular factorial designs had been used.

In order to systematically compare and assess non-regular designs, Deng and Tang (1999) proposed a generalized minimum aberration criterion, a natural generalization of the traditional minimum aberration. This criterion is based on the confounding frequency vector, a concept that generalizes the word-length pattern for regular designs.

Suppose $D$ is a two-level (regular or non-regular) factorial design with $n$ runs and $m$ factors. This design $D$ can also be regarded as a set of $m$ columns $D=\left\{c_{1}, \cdots, c_{m}\right\}$. For any subset of $D$, say, $s=\left\{v_{1}, \cdots, v_{k}\right\}$, define

$$
J_{k}(s)=\left|\sum_{i=1}^{n} v_{i 1} \cdots v_{i k}\right|,
$$

where $v_{i j}$ is the $i^{\text {th }}$ component of column vector $v_{j}$. For example, assume we select 3 columns $v_{1}=\{+1,+1,+1,+1,-1,-1,-1,-1\}^{t}, v_{2}=\{+1,+1,-1,-1,+1,+1,-1,-1\}^{t}$, and $v_{3}=$ $\{+1,-1,+1,-1,+1,-1,+1,-1\}^{t}$ from a design $D$ with 8 runs as a subset $s=\left\{v_{1}, v_{2}, v_{3}\right\}$, where $c^{t}$ is transpose of vector $c$. Then the three-factor-interaction column $v_{1} \cdot v_{2} \cdot v_{3}=$ $\{+1,-1,-1,+1,-1,+1,+1,-1\}^{t}$ is the elementwise product of $v_{1}, v_{2}$, and $v_{3}$, that is, the first element " +1 " of $v_{1} \cdot v_{2} \cdot v_{3}$ is the product of the first elements of $v_{1}, v_{2}$, and $v_{3}$, and second element "- 1 " of $v_{1} \cdot v_{2} \cdot v_{3}$ is the product of the second elements of $v_{1}, v_{2}$, and $v_{3}$, and so on. And then $J_{3}(s)$ is the absolute value of the sum of 8 elements of the interaction column $v_{1} \cdot v_{2} \cdot v_{3}$, so $J_{3}(s)=0$ in this case.

It is easy to check that for regular factorial designs $J_{k}(s)$ equals either 0 or $n$, with 0 corresponding to orthogonality and $n$ to full aliasing. However, for non-regular factorial designs $J_{k}(s)$ is between 0 and $n$. In fact, Deng and Tang $(1999,1998)$ showed that if $n$ is a 
multiple of 8 , then $J_{k}(s)$ is also a multiple of 8 ; and if $n$ is a multiple of 4 but not 8 , then $J_{k}(s)$ is a multiple of 8 for $k=1$ or $2(\bmod 4)$ and a multiple of 8 plus 4 for $k=0 \operatorname{or} 3(\bmod 4)$.

We list all possible values of $J_{k}(s)$ in decreasing order and denote the frequency distribution of $J_{k}(s)$ for all possible $s$ out of $k$ columns in $D$ by $\left[f_{k 11}, f_{k 2}, \cdots, f_{k g+1}\right]$, where $f_{k j}$ is the frequency of $J_{k}(s)$ taking the $j^{t h}$ largest possible value and $g=[n / 8]$, the integer part of $n / 8$. Since $\sum_{j=1}^{y+1} f_{k j}=C(m, k)$, the number of combinations of choosing $k$ out of $m$ factors, it insufficient to consider the first $g$ entries. For example, for a design with 20 runs, $J_{3}(s)$ takes only three possible values $20,12,4$; in this design there are totally 20 (the number of combinations of choosing 3 out 6) possible ways to select out 3 columns; and each possible three-column corresponds to one $J_{3}(s)$ equal to 20 , or 12 , or 4 . Therefore, we only need to record the frequencies of $J_{3}(s)$ equal to 20 , or 12 . Let

$$
F_{k}(D)=\left[f_{k 1}, f_{k 2}, \cdots, f_{k g}\right]
$$

The confounding frequency vector of $D$ is given by

$$
F(D)=\left[F_{3}(D), F_{4}(D), \cdots, F_{m}(D)\right]
$$

since $F_{1}(D)=F_{2}(D)=0$ due to the orthogonality among columns of design.

For any two designs $D_{1}$ and $D_{2}$ with the same run size and the number of factors, let $r$ be the smallest integer such that $F_{r}\left(D_{1}\right) \neq F_{r}\left(D_{2}\right)$. Design $D_{1}$ is said to have less generalized aberration than design $D_{2}$ if $F_{r}\left(D_{1}\right)<F_{r}\left(D_{2}\right)$, which are compared element by element from the first entry to last and the component of $F_{r}\left(D_{1}\right)$ gets small value first. A design is said to have generalized minimum aberration (GMA) if there exists no other design having less generalized aberration. Clearly this criterion reduces to the traditional minimum aberration for regular designs. However, the generalized minimum aberration criterion can compare and assess any two factorial designs no matter whether they are regular or non-regular.

As we know, a generalized minimum aberration design has the "smallest" confounding frequency vector. One simple way to construct the generalized minimum aberration design is to completely search all possible designs and then calculate and compare their confounding frequency vectors. For example, Deng and Tang (1998) studied designs constructed from Hadamard matrices of orders 12 and 16, and Deng, Li and Tang (2000) assessed and compared designs constructed from Hadamard matrices of orders 16, 20 and 24.

In Hadamard matrices, not only any two columns but also any two rows are orthogonal. This property may prevent Hadamard matrices from generating some good designs since the main requirement of factorial designs is the orthogonality among columns. Therefore, Li, Deng and Tang (2000) turned to construct designs directly from orthogonal arrays and found some top designs indeed cannot be embedded into Hadamard matrices.

Using a main effects plan based on a given number of factors and run size, the selected design usually is projected onto low dimension after screening out unimportant factors. It is hoped that the selected design has some other optimal properties, such as, the projected design has higher design efficiency and better model estimability.

For a design $D$ with $n$ runs and $m$ factors, we can check the properties of $X^{t} \times X$, where $X=\left[X_{1}, X_{2}\right], X_{1}$ is the original design $D$ and $X_{2}$ is the $n \times f$ matrix given by 
$f$ two-factor-interactions. The model estimability of a design $D$ can be measured by the vector $\left(I_{1}(D), I_{2}(D), \ldots, I_{F}(D)\right)$, where $I_{f} \quad(1 \leq f \leq F)$, is the number of times that $X^{t} \times X$ is singular when the added $f$ two-factor-interactions go through all $K=C(F, f)$ possible combination choices, and $F=C(m, 2)$ is the total number of possible two-factor-interactions from design $D$.

For example, assume that $D$ is a design with 4 factors, that is $D=X_{1}=\left\{c_{1}, c_{2}, c_{3}, c_{4}\right\}$. There are totally $F=6$ two-factor-interactions from design $D$. If we want to consider the model with four main effects and $f=2$ two-factor-interactions, that is, $Y=\beta_{1} c_{1}+\beta_{2} c_{2}+$ $\beta_{3} c_{3}+\beta_{4} c_{4}+\beta_{5} d_{1}+\beta_{6} d_{2}$, where $d_{1}, d_{2}$ are two different two-factor-interactions, then there are totally $K=15$ (the number of combinations selecting 2 out of 6 total two-factor-interactions) different models and $X_{2}=\left\{d_{1}, d_{2}\right\}$. Therefore, $X=\left[X_{1}, X_{2}\right]$ and $Y=X \beta$. By least square method, we obtained the estimate of $\beta$ as $\left(X^{t} X\right)^{-1} X^{t} Y$ if $X^{t} X$ is non-singular. On the other hand, if $X^{t} X$ is singular, we cannot obtained the estimate of $\beta$ since $\left(X^{t} X\right)^{-1}$ does not exist. We use $I_{2}(D)$ to record the number of times that $X^{t} X$ 's are singular among the 15 models. Therefore, if $I_{f}=0$, then the design can clearly estimate any $f$ two-factor-interactions. Efficiency of a design $D$ can be measured by $D_{f}=\sum_{X_{2}} \operatorname{det}\left(\frac{X^{t} X}{n}\right) / K$, where $(1 \leq f \leq F)$, $X_{2}$ goes through all $K=C(F, f)$ possible models when $f$ two-factor-interactions are added into the main effects model.

In next section, we will compare and assess the top designs with 20 runs that cannot be embedded into Hadamard matrices and those obtained from Hadamard matrices. We show that these new GMA designs are in general superior to the designs obtained from Hadamard matrices when further evaluated by the criteria of model estimability and design efficiency.

\section{$3 \quad$ Results}

Li (2000) provided catalogs of orthogonal arrays with many combinations of different run sizes and the number of factors. For simplicity, we just list several designs that we use in this paper in the following Table 1 , where label $m . k$ means that the orthogonal array with 20 runs has $m$ factors and is ranked $k^{\text {th }}$ in the catalog of non-equivalent orthogonal arrays with 20 runs and $m$ factors, and the star * by the rank indicates that the design cannot be embedded into Hadamard matrices of order 20. Based on the discussion in Section 2 , for designs with the run of $n=20, J_{3}, J_{4}$ and $J_{7}$ take possible values 20, 12, 4. But we only record the frequencies of values 20 and 12 in $F_{3}, F_{4}$ and $F_{7}$. However, it is proved that $J_{k} \neq 20$ and $J_{k} \neq 16$ for $n=20$. Therefore, in this section, $F_{3}, F_{4}$, and $F_{7}$ are the frequencies of $J_{3}=12, J_{4}=12$, and $J_{7}=12$ in the confounding frequency vector, respectively. For similar reason mentioned above, $F_{5}$ and $F_{6}$ are the frequencies of $J_{5}=8$ and $J_{6}=8$ in the confounding frequency vector, respectively.

Designs $6.1 *$ and $7.1 *$ are generalized minimum aberration designs by the GMA criterion. For convenience of recording, we code each run of a design as a binary number by changing level " 1 " as 0 and keeping level " +1 " as 1 . We then convert these binary numbers into common numbers and sort them from smallest to largest. Therefore, a design with $n$ runs and $m$ facotrs can be represented by a sequence of $n$ common numbers between 0 and $2^{m}-1$. 
The integer codes for designs in Table 1 are given in Table 2.

Except for designs $6.1 *, 6.2 *$, and $7.1 *$, other designs in Table 2 can also be obtained from Hadamard matrices of order 20. Designs 6.3 and 7.2 are best designs with 6 or 7 factors obtained from Hadamard matrices, according to GMA criterion. As we know that there are three non-equivalent Hadamard matrices of order 20 (Hall, 1965). For convenience, we list the column numbers for the designs based on the third Hadamard matrix HM3 (see appendix) in Table 3 due to the fact that a complete catalog of non-equivalent designs from three non-equivalent Hadamard matrices can also be obtained from HM3.

Let us take a look at each design from the point of views of design efficiency and model estimability. First, we calculate the design efficiency when $f$ two-factor-interactions are added into the main effects model. When the added $f$ two-factor-interactions go through all possible models, we get the (average) design efficiency recorded in Table 4. For convenience, we only record the design efficiencies for models with 1 to 6 two-factor-interactions. Secondly, for simplicity, we only record the largest $f$ such that $I_{f}=0$ as $I$. I tells the maximum number of two-factor-interactions the design can clearly entertain. For example, we select column 1 to 6 from HM3 as a design (6.34). We add any 3 two-factor-interactions into the main effects model and there are 455 possible models with 3 two-factor-interactions and 6 main effects. This design's efficiency is $D_{3}=0.1408754$ and its $I=3$ indicates each of 455 models can clearly estimate all 6 main effects as well as 3 two-factor-interactions. Table 4 tells us that the designs $6.1^{*}, 6.2^{*}$, and $7.1^{*}$ have higher design efficiencies than other designs. The model estimabilities do not follow the ranking specifically in Table 4. For example, the design 6.1* can clearly estimate at most 6 two-factor-interactions, while design 6.4 can clearly entertain up to 11 two-factor-interactions. However, if we check them from the whole catalog of designs with same runs and same number of factors, the model estimabilities in general match with the GMA ranking. That is, higher ranking design usually has better model estimability. For example, if we happen to select first 6 or 7 columns from HM3 (after dropping column 0) as a design with 6 or 7 factors, they are lowest ranking designs (6.34 and 7.71) and they have lowest design efficiencies and worst model estimability.

If not every main effect is significant, we usually project the design onto a lower dimension. We wish to select a design with the property that the projected designs also have the high design efficiency and good model estimability. Design efficiency and model estimability of projections from 6 factors onto 5 factors and 4 factors are recorded in Table 5 and Table 6 , respectively, where freq indicates the number of projections having the same design efficiency and model estimability. From Tables 5 and 6 , we see that designs $6.1^{*}$ and $6.2^{*}$ have higher design efficiencies and better model estimability. For example, the projections onto 5 columns from designs $6.1^{*}$ and $6.2^{*}$ have almost uniformly higher design efficiencies, while there are at least two out of six projections from other designs having lower design efficiencies. Any projection onto 4 columns can estimate all 6 two-factor-interactions plus 4 main effects. All 15 projections onto 4 columns from designs $6.1^{*}$ and $6.2^{*}$ have uniformly highest design efficiencies, and projections from other designs may have much lower design efficiency. Again, if we happen to choose the first 6 columns as a design (6.34), their projections always have lowest design efficiency and worst model estimability.

Therefore, if we are mainly interested in main effects, plus a few two-factor-interactions, 
and design efficiency is a major concern; or if we know that not all 6 factors are significant, then designs $6.1^{*}$ and $6.2^{*}$ are the best candidates. On the other hand, if the design efficiency is not a problem (e.g. some industrial experiments can be controlled to have little error), and we are interested in all main effects and as many as possible two-factor-interactions, then design 6.4 is a better choice.

For designs of 20 runs and 7 factors, we can draw a very similar conclusion in that GMA design 7.1* has higher design efficiency and better model estimability. Design 7.1* is a better candidate for an experiment in which we are interested in main effects and twofactor-interactions as well as design efficiency.

\section{Summary}

In this paper, we study top orthogonal arrays that cannot be embedded into Hadamad matrices from the angle of projection. We show that these new GMA orthogonal arrays are also superior to the best designs obtained from Hadamard matrices when their projections are further evaluated by the criteria of model estimability and design efficiency.

\section{References}

[1] Deng, L.Y., Li, Y., and Tang, B. (2000). Catalogue of non-regular designs with small runs from Hadamard matrices based on generalized minimum aberration criterion. Comm. Statist. Theory Methods 29, 1379-1395.

[2] Deng, L.Y., and Tang, B. (1999). Generalized resolution and minimum aberration criteria for Plackett-Burman and other non-regular designs. Statistica Sinica 9, 1071-1082.

[3] Fries, A. and Hunter, W.G. (1980). Minimum aberration $2^{k-p}$ designs. Technometrics 22, 601-608.

[4] Hall, M.J. (1965). Hadamard Matrices of order 20. Jet Propulsion Laboratory, Technical Report 32-76.

[5] Hamada, M. and Wu, C.F.J. (1992). Analysis of designed experiments with complex aliasing. Journal of Quality Technology 24, 130-137.

[6] Li, Y. Deng, L.Y., and Tang, B. (2000). Computer generation of non-equivalent orthogonal arrays and generalized minimum aberration designs. Submitted for publication.

[7] Spence, E. (1995). Classification of Hadamard matrices of order 24 and 28. Discrete Mathematics 140, 185-243. 
[8] Vijayan, K. (1976). Hadamard Matrices and Submatrices. J. Austral. Math. Soc. 22A, 469-475.

\section{Appendix}

Table 1: Rankings and Confounding Frequency Vectors for designs with 20 runs

\begin{tabular}{|l|rrrrr|}
\hline Rank & $F_{3}$ & $F_{4}$ & $F_{5}$ & $F_{6}$ & $F_{7}$ \\
\hline $6.1^{*}$ & 0 & 0 & 4 & 1 & \\
$6.2^{*}$ & 0 & 0 & 5 & 0 & \\
6.3 & 0 & 1 & 2 & 1 & \\
6.4 & 0 & 1 & 3 & 0 & \\
6.5 & 0 & 2 & 1 & 0 & \\
6.42 & 4 & 3 & 3 & 0 & \\
\hline $7.1^{*}$ & 0 & 2 & 11 & 1 & 0 \\
7.2 & 0 & 3 & 7 & 3 & 0 \\
7.3 & 0 & 3 & 9 & 1 & 0 \\
7.4 & 0 & 4 & 5 & 3 & 0 \\
7.5 & 0 & 4 & 7 & 1 & 0 \\
7.71 & 7 & 7 & 9 & 3 & 0 \\
\hline
\end{tabular}

Table 2: Designs and their corresponding codes

\begin{tabular}{|l|r|c|}
\hline Design & Code for Run \\
\hline $6.1^{*}$ & 127814192025283136374142474851545861 \\
$6.2^{*}$ & 037814182125283136374143464851545861 \\
6.3 & 0121215212226272937394043464851545760 \\
6.4 & 0121314202325263136394143465051535660 \\
6.5 & 0121314202325273037384043475051535660 \\
6.42 & 33448232626292939414146464849505263 \\
\hline $7.1^{*}$ & 251516293841515662737483849496103108117123 \\
7.2 & 124272841465055617577808794100103106113120 \\
7.3 & 124272840475154617477818794101102107112120 \\
7.4 & 124262941475154607576808795101102106113120 \\
7.5 & 0472526424551546175768187949798111116120 \\
7.71 & 669916475353585879838392929798100104127 \\
\hline
\end{tabular}


Table 3: Designs and their corresponding columns from HM3

\begin{tabular}{|l|rrrrrrr|}
\hline Design & \multicolumn{7}{l|}{ Corresponding columns } \\
\hline 6.3 & 1 & 2 & 4 & 7 & 12 & 18 & \\
6.4 & 1 & 2 & 4 & 7 & 8 & 16 & \\
6.5 & 1 & 2 & 4 & 7 & 8 & 12 & \\
6.42 & 1 & 2 & 3 & 4 & 5 & 6 & \\
\hline 7.2 & 1 & 2 & 4 & 7 & 8 & 12 & 18 \\
7.3 & 1 & 2 & 4 & 7 & 8 & 12 & 16 \\
7.4 & 1 & 2 & 4 & 7 & 8 & 12 & 15 \\
7.5 & 1 & 2 & 4 & 7 & 10 & 12 & 17 \\
7.71 & 1 & 2 & 3 & 4 & 5 & 6 & 7 \\
\hline
\end{tabular}

Table 4: Designs and their design efficiency and model estimability

\begin{tabular}{|l|lllllll|}
\hline Rank & $f=1$ & $f=2$ & $f=3$ & $f=4$ & $f=5$ & $f=6$ & $I$ \\
\hline $6.1^{*}$ & 0.84 & 0.6838857 & 0.5379578 & 0.4073439 & 0.2955829 & 0.204407 & 6 \\
$6.2^{*}$ & 0.84 & 0.6851048 & 0.5404469 & 0.410421 & 0.2983218 & 0.2060656 & 9 \\
6.3 & 0.84 & 0.6760838 & 0.5193773 & 0.3790878 & 0.2614436 & 0.1692147 & 9 \\
6.4 & 0.84 & 0.6773029 & 0.5220149 & 0.3825658 & 0.2648278 & 0.1716673 & 11 \\
6.5 & 0.84 & 0.6699886 & 0.5044562 & 0.3559964 & 0.2333675 & 0.1404592 & 9 \\
6.42 & 0.584 & 0.3064686 & 0.1408754 & 0.054526 & 0.0166398 & 0.0035355 & 3 \\
\hline $7.1^{*}$ & 0.8 & 0.6089143 & 0.4380934 & 0.2955392 & 0.1850405 & 0.1061121 & 5 \\
7.2 & 0.8 & 0.604221 & 0.4280921 & 0.2822535 & 0.1714216 & 0.094626 & 5 \\
7.3 & 0.8 & 0.6058057 & 0.4313735 & 0.2864791 & 0.1756154 & 0.0980504 & 5 \\
7.4 & 0.8 & 0.6011124 & 0.4213044 & 0.2731391 & 0.1621145 & 0.0869272 & 5 \\
7.5 & 0.8 & 0.6026971 & 0.4246428 & 0.2774673 & 0.1663903 & 0.0903616 & 5 \\
7.71 & 0.48 & 0.2011429 & 0.0708331 & 0.0197101 & 0.0038647 & 0.0004019 & 3 \\
\hline
\end{tabular}

Table 5: Design efficiency and model estimability of projections onto 5 columns

\begin{tabular}{|c|llllllll|}
\hline Design & $f=1$ & $f=2$ & $f=3$ & $f=4$ & $f=5$ & $f=6$ & freq & 1 \\
\hline \multirow{2}{*}{$6.1^{*}$} & 0.88 & 0.7588978 & 0.6403413 & 0.5277115 & 0.4239022 & 0.3311439 & 4 & 10 \\
& 0.88 & 0.7573333 & 0.636928 & 0.5232581 & 0.4199097 & 0.3292768 & 2 & 10 \\
\hline \multirow{2}{*}{$6.2^{*}$} & 0.88 & 0.7588978 & 0.6403413 & 0.5277115 & 0.4239022 & 0.3311439 & 5 & 10 \\
& 0.88 & 0.7573333 & 0.636928 & 0.5232581 & 0.4199097 & 0.3292768 & 1 & 10 \\
\hline \multirow{3}{*}{6.3} & 0.88 & 0.7588978 & 0.6403413 & 0.5277115 & 0.4239022 & 0.3311439 & 2 & 10 \\
& 0.88 & 0.7573333 & 0.636928 & 0.5232581 & 0.4199097 & 0.3292768 & 2 & 10 \\
& 0.88 & 0.7391289 & 0.5888683 & 0.4415753 & 0.3085909 & 0.1984397 & 2 & 10 \\
\hline \multirow{6}{*}{6.4} & 0.88 & 0.7588978 & 0.6403413 & 0.5277115 & 0.4239022 & 0.3311439 & 3 & 10 \\
& 0.88 & 0.7573333 & 0.636928 & 0.5232581 & 0.4199097 & 0.3292768 & 1 & 10 \\
& 0.88 & 0.7391289 & 0.5888683 & 0.4415753 & 0.3085909 & 0.1984397 & 2 & 10 \\
\hline \multirow{6}{*}{6.5} & 0.88 & 0.7573333 & 0.636928 & 0.5232581 & 0.4199097 & 0.3292768 & 2 & 10 \\
& 0.88 & 0.7418311 & 0.5946027 & 0.4485534 & 0.3138455 & 0.1992111 & 1 & 5 \\
& 0.88 & 0.7391289 & 0.5888683 & 0.4415753 & 0.3085909 & 0.1984397 & 3 & 10 \\
\hline \multirow{2}{*}{6.34} & 0.688 & 0.4357689 & 0.2495829 & 0.1257293 & 0.0530946 & 0.0170648 & 3 & 3 \\
& 0.688 & 0.4353422 & 0.2499243 & 0.1273021 & 0.0552117 & 0.0186882 & 3 & 3 \\
\hline
\end{tabular}




\section{Applied Statistics in Agriculture}

Table 6: Design efficiency and model estimability of projections onto 4 columns

\begin{tabular}{|c|lllllll|}
\hline Design & $f=1$ & $f=2$ & $f=3$ & $f=4$ & $f=5$ & $f=6$ & freq \\
\hline $6.1^{*}$ & 0.92 & 0.83712 & 0.75264 & 0.6679429 & 0.58445 & 0.5035262 & 15 \\
\hline $6.2^{*}$ & 0.92 & 0.83712 & 0.75264 & 0.6679429 & 0.58445 & 0.5035262 & 15 \\
\hline \multirow{2}{*}{6.3} & 0.92 & 0.83712 & 0.75264 & 0.6679429 & 0.58445 & 0.5035262 & 14 \\
& 0.92 & 0.77312 & 0.577536 & 0.3743416 & 0.2160067 & 0.1140851 & 1 \\
\hline \multirow{2}{*}{6.4} & 0.92 & 0.83712 & 0.75264 & 0.6679429 & 0.58445 & 0.5035262 & 14 \\
& 0.92 & 0.77312 & 0.577536 & 0.3743416 & 0.2160067 & 0.1140851 & 1 \\
\hline \multirow{2}{*}{6.5} & 0.92 & 0.83712 & 0.75264 & 0.6679429 & 0.58445 & 0.5035262 & 13 \\
& 0.92 & 0.77312 & 0.577536 & 0.3743416 & 0.2160067 & 0.1140851 & 2 \\
\hline \multirow{2}{*}{6.34} & 0.92 & 0.77312 & 0.577536 & 0.3743416 & 0.2160067 & 0.1140851 & 3 \\
& 0.76 & 0.55808 & 0.395264 & 0.2700083 & 0.1782579 & 0.1140851 & 12 \\
\hline
\end{tabular}

Table 7: The third Hadamard matrix HM3 of order 20

\begin{tabular}{|rrrrrrrrrrrrrrrrrrrr|}
\hline 0 & 1 & 2 & 3 & 4 & 5 & 6 & 7 & 8 & 9 & 10 & 11 & 12 & 13 & 14 & 15 & 16 & 17 & 18 & 19 \\
\hline 1 & 1 & 1 & 1 & 1 & 1 & 1 & 1 & -1 & -1 & -1 & -1 & -1 & -1 & -1 & -1 & -1 & -1 & -1 & -1 \\
1 & 1 & 1 & -1 & 1 & -1 & -1 & -1 & -1 & -1 & 1 & 1 & -1 & -1 & 1 & 1 & -1 & -1 & 1 & 1 \\
1 & 1 & 1 & -1 & -1 & 1 & -1 & -1 & 1 & 1 & -1 & -1 & 1 & 1 & -1 & -1 & -1 & -1 & 1 & 1 \\
1 & 1 & 1 & -1 & -1 & -1 & 1 & -1 & 1 & 1 & -1 & -1 & -1 & -1 & 1 & 1 & 1 & 1 & -1 & -1 \\
1 & 1 & 1 & -1 & -1 & -1 & -1 & 1 & -1 & -1 & 1 & 1 & 1 & 1 & -1 & -1 & 1 & 1 & -1 & -1 \\
1 & 1 & -1 & -1 & 1 & 1 & 1 & 1 & 1 & 1 & 1 & 1 & 1 & 1 & 1 & 1 & -1 & -1 & -1 & -1 \\
1 & 1 & -1 & 1 & 1 & 1 & -1 & -1 & 1 & -1 & 1 & -1 & 1 & -1 & -1 & 1 & 1 & 1 & 1 & -1 \\
1 & 1 & -1 & 1 & 1 & 1 & -1 & -1 & -1 & 1 & -1 & 1 & -1 & 1 & 1 & -1 & 1 & 1 & -1 & 1 \\
1 & 1 & -1 & 1 & -1 & -1 & 1 & 1 & 1 & -1 & 1 & -1 & -1 & 1 & 1 & -1 & 1 & -1 & 1 & 1 \\
1 & 1 & -1 & 1 & -1 & -1 & 1 & 1 & -1 & 1 & -1 & 1 & 1 & -1 & -1 & 1 & -1 & 1 & 1 & 1 \\
1 & -1 & 1 & -1 & 1 & 1 & 1 & 1 & 1 & 1 & 1 & 1 & -1 & -1 & -1 & -1 & 1 & 1 & 1 & 1 \\
1 & -1 & 1 & 1 & 1 & -1 & 1 & -1 & 1 & -1 & -1 & 1 & 1 & 1 & 1 & -1 & -1 & 1 & 1 & -1 \\
1 & -1 & 1 & 1 & 1 & -1 & 1 & -1 & -1 & 1 & 1 & -1 & 1 & 1 & -1 & 1 & 1 & -1 & -1 & 1 \\
1 & -1 & 1 & 1 & -1 & 1 & -1 & 1 & 1 & -1 & -1 & 1 & 1 & -1 & 1 & 1 & 1 & -1 & -1 & 1 \\
1 & -1 & 1 & 1 & -1 & 1 & -1 & 1 & -1 & 1 & 1 & -1 & -1 & 1 & 1 & 1 & -1 & 1 & 1 & -1 \\
1 & -1 & -1 & 1 & -1 & -1 & -1 & -1 & 1 & 1 & 1 & 1 & -1 & -1 & -1 & -1 & -1 & -1 & -1 & -1 \\
1 & -1 & -1 & -1 & 1 & -1 & -1 & 1 & 1 & -1 & -1 & -1 & -1 & 1 & -1 & 1 & -1 & 1 & -1 & 1 \\
1 & -1 & -1 & -1 & 1 & -1 & -1 & 1 & -1 & 1 & -1 & -1 & 1 & -1 & 1 & -1 & 1 & -1 & 1 & -1 \\
1 & -1 & -1 & -1 & -1 & 1 & 1 & -1 & -1 & -1 & 1 & -1 & 1 & -1 & 1 & -1 & -1 & 1 & -1 & 1 \\
1 & -1 & -1 & -1 & -1 & 1 & 1 & -1 & -1 & -1 & -1 & 1 & -1 & 1 & -1 & 1 & 1 & -1 & 1 & -1 \\
\hline
\end{tabular}


Table 8: Design efficiency and model estimability of projections onto 6 columns

\begin{tabular}{|c|c|c|c|c|c|c|c|c|}
\hline Design & $f=1$ & $f=2$ & $f=3$ & $f=4$ & $f=5$ & $f=6$ & freq & I \\
\hline \multirow{4}{*}{$7.1^{*}$} & 0.84 & 0.6851048 & 0.5404469 & 0.410421 & 0.2983218 & 0.2060656 & 1 & 9 \\
\hline & 0.84 & 0.6838857 & 0.5379578 & 0.4073439 & 0.2955829 & 0.204407 & 1 & 6 \\
\hline & 0.84 & 0.6777905 & 0.5230682 & 0.3840443 & 0.2665376 & 0.1734532 & 4 & 5 \\
\hline & 0.84 & 0.669501 & 0.5033669 & 0.3544888 & 0.2317712 & 0.1390879 & 1 & 9 \\
\hline \multirow{3}{*}{7.2} & 0.84 & 0.6773029 & 0.5220149 & 0.3825658 & 0.2648278 & 0.1716673 & 2 & 11 \\
\hline & 0.84 & 0.6760838 & 0.5193773 & 0.3790878 & 0.2614436 & 0.1692147 & 3 & 9 \\
\hline & 0.84 & 0.6699886 & 0.5044562 & 0.3559964 & 0.2333675 & 0.1404592 & 2 & 5 \\
\hline \multirow{5}{*}{7.3} & 0.84 & 0.6777905 & 0.5230682 & 0.3840443 & 0.2665376 & 0.1734532 & 2 & 5 \\
\hline & 0.84 & 0.6773029 & 0.5220149 & 0.3825658 & 0.2648278 & 0.1716673 & 2 & 11 \\
\hline & 0.84 & 0.677059 & 0.5214613 & 0.3818411 & 0.2641921 & 0.1713531 & 1 & 5 \\
\hline & 0.84 & 0.6710857 & 0.5065964 & 0.3584032 & 0.2351371 & 0.1410647 & 1 & 5 \\
\hline & 0.84 & 0.6699886 & 0.5044562 & 0.3559964 & 0.2333675 & 0.1404592 & 1 & 5 \\
\hline \multirow{5}{*}{7.4} & 0.84 & 0.677059 & 0.5214613 & 0.3818411 & 0.2641921 & 0.1713531 & 1 & 5 \\
\hline & 0.84 & 0.6760838 & 0.5193773 & 0.3790878 & 0.2614436 & 0.1692147 & 2 & 9 \\
\hline & 0.84 & 0.6699886 & 0.5044562 & 0.3559964 & 0.2333675 & 0.1404592 & 1 & 5 \\
\hline & 0.84 & 0.669501 & 0.5033669 & 0.3544888 & 0.2317712 & 0.1390879 & 2 & 9 \\
\hline & 0.84 & 0.6632838 & 0.4878809 & 0.3305194 & 0.2032821 & 0.1114051 & 1 & 5 \\
\hline \multirow{5}{*}{7.5} & 0.84 & 0.6777905 & 0.5230682 & 0.3840443 & 0.2665376 & 0.1734532 & 2 & 5 \\
\hline & 0.84 & 0.677059 & 0.5214613 & 0.3818411 & 0.2641921 & 0.1713531 & 1 & 5 \\
\hline & 0.84 & 0.6710857 & 0.5065964 & 0.3584032 & 0.2351371 & 0.1410647 & 1 & 5 \\
\hline & 0.84 & 0.6699886 & 0.5044562 & 0.3559964 & 0.2333675 & 0.1404592 & 2 & 5 \\
\hline & 0.84 & 0.6632838 & 0.4878809 & 0.3305194 & 0.2032821 & 0.1114051 & 1 & 5 \\
\hline \multirow{2}{*}{7.71} & 0.584 & 0.3064686 & 0.1408754 & 0.054526 & 0.0166398 & 0.0035355 & 4 & 3 \\
\hline & 0.584 & 0.303299 & 0.1365723 & 0.0513457 & 0.0151561 & 0.0031279 & 3 & 3 \\
\hline
\end{tabular}


Table 9: Design efficiency and model estimability of projections onto 5 columns

\begin{tabular}{|c|llllllll|}
\hline Design & $f=1$ & $f=2$ & $f=3$ & $f=4$ & $f=5$ & $f=6$ & freq & $I$ \\
\hline \multirow{7}{*}{$7.1^{*}$} & 0.88 & 0.7588978 & 0.6403413 & 0.5277115 & 0.4239022 & 0.3311439 & 9 & 10 \\
& 0.88 & 0.7573333 & 0.636928 & 0.5232581 & 0.4199097 & 0.3292768 & 6 & 10 \\
& 0.88 & 0.7418311 & 0.5946027 & 0.4485534 & 0.3138455 & 0.1992111 & 2 & 5 \\
& 0.88 & 0.7391289 & 0.5888683 & 0.4415753 & 0.3085909 & 0.1984397 & 4 & 10 \\
\hline \multirow{7}{*}{7.2} & 0.88 & 0.7588978 & 0.6403413 & 0.5277115 & 0.4239022 & 0.3311439 & 6 & 10 \\
& 0.88 & 0.7573333 & 0.636928 & 0.5232581 & 0.4199097 & 0.3292768 & 6 & 10 \\
& 0.88 & 0.7418311 & 0.5946027 & 0.4485534 & 0.3138455 & 0.1992111 & 1 & 5 \\
& 0.88 & 0.7391289 & 0.5888683 & 0.4415753 & 0.3085909 & 0.1984397 & 8 & 10 \\
\hline \multirow{7}{*}{7.3} & 0.88 & 0.7588978 & 0.6403413 & 0.5277115 & 0.4239022 & 0.3311439 & 6 & 10 \\
& 0.88 & 0.7573333 & 0.636928 & 0.5232581 & 0.4199097 & 0.3292768 & 6 & 10 \\
& 0.88 & 0.7418311 & 0.5946027 & 0.4485534 & 0.3138455 & 0.1992111 & 3 & 5 \\
& 0.88 & 0.7391289 & 0.5888683 & 0.4415753 & 0.3085909 & 0.1984397 & 6 & 10 \\
\hline \multirow{7}{*}{7.4} & 0.88 & 0.7588978 & 0.6403413 & 0.5277115 & 0.4239022 & 0.3311439 & 3 & 10 \\
& 0.88 & 0.7573333 & 0.636928 & 0.5232581 & 0.4199097 & 0.3292768 & 6 & 10 \\
& 0.88 & 0.7418311 & 0.5946027 & 0.4485534 & 0.3138455 & 0.1992111 & 2 & 5 \\
& 0.88 & 0.7391289 & 0.5888683 & 0.4415753 & 0.3085909 & 0.1984397 & 10 & 10 \\
\hline \multirow{7}{*}{7.5} & 0.88 & 0.7588978 & 0.6403413 & 0.5277115 & 0.4239022 & 0.3311439 & 3 & 10 \\
& 0.88 & 0.7573333 & 0.636928 & 0.5232581 & 0.4199097 & 0.3292768 & 6 & 10 \\
& 0.88 & 0.7418311 & 0.5946027 & 0.4485534 & 0.3138455 & 0.1992111 & 4 & 5 \\
& 0.88 & 0.7391289 & 0.5888683 & 0.4415753 & 0.3085909 & 0.1984397 & 8 & 10 \\
\hline \multirow{2}{*}{7.71} & 0.688 & 0.4357689 & 0.2495829 & 0.1257293 & 0.0530946 & 0.0170648 & 9 & 3 \\
& 0.688 & 0.4353422 & 0.2499243 & 0.1273021 & 0.0552117 & 0.0186882 & 12 & 3 \\
\hline
\end{tabular}

Table 10: Design efficiency and model estimability of projections onto 4 columns

\begin{tabular}{|c|lllllll|}
\hline Design & $f=1$ & $f=2$ & $f=3$ & $f=4$ & $f=5$ & $f=6$ & freq \\
\hline \multirow{2}{*}{$7.1^{*}$} & 0.92 & 0.83712 & 0.75264 & 0.6679429 & 0.58445 & 0.5035262 & 33 \\
& 0.92 & 0.77312 & 0.577536 & 0.3743416 & 0.2160067 & 0.1140851 & 2 \\
\hline \multirow{2}{*}{7.2} & 0.92 & 0.83712 & 0.75264 & 0.6679429 & 0.58445 & 0.5035262 & 32 \\
& 0.92 & 0.77312 & 0.577536 & 0.3743416 & 0.2160067 & 0.1140851 & 3 \\
\hline \multirow{2}{*}{7.3} & 0.92 & 0.83712 & 0.75264 & 0.6679429 & 0.58445 & 0.5035262 & 32 \\
& 0.92 & 0.77312 & 0.577536 & 0.3743416 & 0.2160067 & 0.1140851 & 3 \\
\hline \multirow{2}{*}{7.4} & 0.92 & 0.83712 & 0.75264 & 0.6679429 & 0.58445 & 0.5035262 & 31 \\
& 0.92 & 0.77312 & 0.577536 & 0.3743416 & 0.2160067 & 0.1140851 & 4 \\
\hline \multirow{2}{*}{7.5} & 0.92 & 0.83712 & 0.75264 & 0.6679429 & 0.58445 & 0.5035262 & 31 \\
& 0.92 & 0.77312 & 0.577536 & 0.3743416 & 0.2160067 & 0.1140851 & 4 \\
\hline \multirow{2}{*}{7.71} & 0.92 & 0.77312 & 0.577536 & 0.3743416 & 0.2160067 & 0.1140851 & 7 \\
& 0.76 & 0.55808 & 0.395264 & 0.2700083 & 0.1782579 & 0.1140851 & 28 \\
\hline
\end{tabular}


Table 11: Design 6.1*

\begin{tabular}{|rrrrrr|}
\hline 1 & 2 & 3 & 4 & 5 & 6 \\
\hline 1 & 1 & 1 & 1 & -1 & 1 \\
1 & 1 & 1 & -1 & 1 & -1 \\
1 & 1 & -1 & -1 & -1 & -1 \\
1 & 1 & -1 & -1 & 1 & 1 \\
1 & 1 & -1 & 1 & 1 & -1 \\
1 & -1 & 1 & 1 & 1 & 1 \\
1 & -1 & 1 & -1 & -1 & 1 \\
1 & -1 & 1 & -1 & 1 & -1 \\
1 & -1 & -1 & 1 & -1 & -1 \\
1 & -1 & -1 & 1 & -1 & 1 \\
-1 & 1 & 1 & 1 & -1 & -1 \\
-1 & 1 & 1 & 1 & 1 & 1 \\
-1 & 1 & 1 & -1 & -1 & 1 \\
-1 & 1 & -1 & -1 & 1 & 1 \\
-1 & 1 & -1 & 1 & -1 & -1 \\
-1 & -1 & 1 & 1 & 1 & -1 \\
-1 & -1 & 1 & -1 & -1 & -1 \\
-1 & -1 & -1 & -1 & 1 & -1 \\
-1 & -1 & -1 & -1 & -1 & 1 \\
-1 & -1 & -1 & 1 & 1 & 1 \\
\hline
\end{tabular}

Table 12: Design 7.1*

\begin{tabular}{|rrrrrrr|}
\hline 1 & 2 & 3 & 4 & 5 & 6 & 7 \\
\hline 1 & 1 & 1 & 1 & -1 & 1 & 1 \\
1 & 1 & 1 & -1 & 1 & -1 & 1 \\
1 & 1 & -1 & -1 & -1 & -1 & -1 \\
1 & 1 & -1 & -1 & 1 & 1 & 1 \\
1 & 1 & -1 & 1 & 1 & -1 & -1 \\
1 & -1 & 1 & 1 & 1 & 1 & -1 \\
1 & -1 & 1 & -1 & -1 & 1 & 1 \\
1 & -1 & 1 & -1 & 1 & -1 & -1 \\
1 & -1 & -1 & 1 & -1 & -1 & 1 \\
1 & -1 & -1 & 1 & -1 & 1 & -1 \\
-1 & 1 & 1 & 1 & -1 & -1 & -1 \\
-1 & 1 & 1 & 1 & 1 & 1 & -1 \\
-1 & 1 & 1 & -1 & -1 & 1 & 1 \\
-1 & 1 & -1 & -1 & 1 & 1 & -1 \\
-1 & 1 & -1 & 1 & -1 & -1 & 1 \\
-1 & -1 & 1 & 1 & 1 & -1 & 1 \\
-1 & -1 & 1 & -1 & -1 & -1 & -1 \\
-1 & -1 & -1 & -1 & 1 & -1 & 1 \\
-1 & -1 & -1 & -1 & -1 & 1 & -1 \\
-1 & -1 & -1 & 1 & 1 & 1 & 1 \\
\hline
\end{tabular}

